\title{
Preoperative depression, lumbar fusion, and opioid use: an assessment of postoperative prescription, quality, and economic outcomes
}

\author{
Chloe O'Connell, BS, ${ }^{1}$ Tej Deepak Azad, MS, ${ }^{2}$ Vaishali Mittal, BS, ${ }^{1}$ Daniel Vail, BA, ${ }^{1}$ \\ Eli Johnson, BS, ${ }^{2}$ Atman Desai, MD, ${ }^{2}$ Eric Sun, MD, PhD, ${ }^{3}$ John K. Ratliff, MD, ${ }^{2}$ and \\ Anand Veeravagu, MD²
}

Departments of ${ }^{2}$ Neurosurgery and ${ }^{3}$ Anesthesiology, Perioperative and Pain Medicine, ${ }^{1}$ Stanford University School of Medicine, Stanford, California

OBJECTIVE Preoperative depression has been linked to a variety of adverse outcomes following lumbar fusion, including increased pain, disability, and 30-day readmission rates. The goal of the present study was to determine whether preoperative depression is associated with increased narcotic use following lumbar fusion. Moreover, the authors examined the association between preoperative depression and a variety of secondary quality indicator and economic outcomes, including complications, 30-day readmissions, revision surgeries, likelihood of discharge home, and 1- and 2-year costs.

METHODS A retrospective analysis was conducted using a national longitudinal administrative database (MarketScan) containing diagnostic and reimbursement data on patients with a variety of private insurance providers and Medicare for the period from 2007 to 2014. Multivariable logistic and negative binomial regressions were performed to assess the relationship between preoperative depression and the primary postoperative opioid use outcomes while controlling for demographic, comorbidity, and preoperative prescription drug-use variables. Logistic and log-linear regressions were also used to evaluate the association between depression and the secondary outcomes of complications, 30-day readmissions, revisions, likelihood of discharge home, and 1- and 2-year costs.

RESULTS The authors identified 60,597 patients who had undergone lumbar fusion and met the study inclusion criteria, 4985 of whom also had a preoperative diagnosis of depression and 21,905 of whom had a diagnosis of spondylolisthesis at the time of surgery. A preoperative depression diagnosis was associated with increased cumulative opioid use $(\beta=$ $0.25, p<0.001$ ), an increased risk of chronic use (OR 1.28, 95\% CI 1.17-1.40), and a decreased probability of opioid cessation (OR $0.96,95 \% \mathrm{Cl} 0.95-0.98$ ) following lumbar fusion. In terms of secondary outcomes, preoperative depression was also associated with a slightly increased risk of complications (OR 1.14, 95\% Cl 1.03-1.25), revision fusions (OR 1.15, 95\% Cl 1.05-1.26), and 30-day readmissions (OR 1.19, 95\% Cl 1.04-1.36), although it was not significantly associated with the probability of discharge to home (OR $0.92,95 \% \mathrm{Cl} 0.84-1.01)$. Preoperative depression also resulted in increased costs at $1(\beta=0.06, p<0.001)$ and $2(\beta=0.09, p<0.001)$ years postoperatively.

CONCLUSIONS Although these findings must be interpreted in the context of the limitations inherent to retrospective studies utilizing administrative data, they provide additional evidence for the link between a preoperative diagnosis of depression and adverse outcomes, particularly increased opioid use, following lumbar fusion.

https://thejns.org/doi/abs/10.3171/2017.10.FOCUS17563

KEY WORDS opioids; lumbar fusion; depression

ABBREVIATIONS $\mathrm{CDC}=$ Centers for Disease Control and Prevention; $\mathrm{CPT}=$ Current Procedural Terminology; $\mathrm{MME}=$ morphine milligram equivalent .

SUBMITTED August 31, 2017. ACCEPTED October 23, 2017.

INCLUDE WHEN CITING DOI: 10.3171/2017.10.FOCUS17563. 
$\mathrm{M}$ AJOR depressive disorder is the leading cause of disability among people between the ages of 15 and 44 years, with a 12 -month prevalence of $6.6 \%$ and a lifetime prevalence of $16.2 \% .^{7,16}$ The relationship between depression and low-back pain is well established, ${ }^{7}$ with depressive symptomatology linked to a more than 2-fold increase in the odds of low-back pain in 1 study. ${ }^{25}$ Despite this association, as well as the link between depression and adverse outcomes after many types of surgery, ${ }^{5,21}$ the impact of depression on outcomes following lumbar fusion has yet to be fully characterized.

Previous studies have described associations between preoperative depression and various outcomes of spinal surgery, including postoperative disability, patient satisfaction, and 30-day readmission rates. ${ }^{1-3,23,29}$ Notably, the depressed population is particularly vulnerable to substance abuse and dependence issues, ${ }^{7,17}$ but the link between depression and opioid use and abuse following lumbar fusion has remained largely unexplored. The only study reporting a link between depression and greater opioid use after lumbar fusion was performed in a workers' compensation setting, which has been associated with poorer overall outcomes than those of the general population, limiting the external validity and applicability of these findings. ${ }^{6,14,22}$ The association between depression and opioid use following fusion is increasingly relevant given the epidemic of opioid abuse sweeping the country in recent years. ${ }^{15,30,34,35}$

In the present study, we used data from the MarketScan national administrative database (Truven Health Analytics) to examine the effects of preoperative depression on opioid use following lumbar fusion, controlling for different levels of preoperative opioid use and other confounding variables. Secondarily, we examined whether patients with a preexisting diagnosis of depression have poorer overall lumbar fusion outcomes than the patients without depression. A variety of different quality indicator and economic outcomes were considered, including complications, 30-day all-cause readmissions, revision surgeries, discharge home, and 1- and 2-year costs.

\section{Methods \\ Data Source}

To conduct this study, we queried an observational administrative database of patients in the United States who had undergone lumbar fusion between 2007 and 2014. Utilization data in the inpatient, outpatient, and pharmacy settings were obtained from the MarketScan Commercial Claims and Encounters Database and Medicare Supplemental and Coordination of Benefits Database. These data encompass health care claims submitted on behalf of individuals enrolled in private insurance plans and Medicare through a participating employer, health plan, or government organization. Both inpatient and outpatient data, such as diagnosis, date of service, demographics, and employer information, were reviewed. The linked drug prescription database included information on all prescriptions covered by insurance that were filled by the patient, along with dosage, drug identification number, day supply, and prescription date. The data are frequently used in analyses of health care utilization and spending. ${ }^{18,31,33,36}$

\section{Patient Sample}

We obtained our study cohort by utilizing Current Procedural Terminology (CPT) codes to identify patients older than 18 years who had undergone lumbar fusion between 2007 and 2014. For a full list of codes used to define lumbar fusion, see Table 1. For each patient, we defined the "index date" as the date of the first fusion surgery within the coverage period and the "index visit" as the inpatient visit in which that surgery took place. We restricted our analysis to patients who were continuously enrolled in a health insurance plan offering pharmacy and medical benefits for the period 6 months before the index date and at least 2 years after the index date, meaning that they had to have been alive and actively enrolled in a participating plan for a minimum of 2 years following lumbar fusion. This effectively limited our sample to patients who had undergone lumbar fusion between July 1, 2007, and December 31, 2012. To restrict our sample to patients who had undergone elective surgery for degenerative disease and to limit confounding from previous surgery, we excluded those with International Classification of Diseases, Ninth Revision (ICD-9) codes for a preoperative diagnosis of trauma, postlaminectomy syndrome, systemic malignancy, paralysis, schizophrenia, and bipolar disorder.

\section{Outcomes}

The primary outcomes used to assess opioid use in the year following surgery were total cumulative dose, chronic use, and cessation of opioids. Total cumulative dose in morphine milligram equivalents (MMEs) 3-12 months post-index visit was calculated by multiplying the total quantity of pills by the strength of the prescription (a number representing the concentration of the active opioid ingredient), and multiplying that product by the MME conversion factor provided by the Centers for Disease Control and Prevention (CDC). ${ }^{26}$ This allowed for comparison across opioid types in the form of MMEs (for reference, $50 \mathrm{mg}$ of hydrocodone translates to 50 MMEs, and the CDC recommends avoiding or carefully monitoring a prescription > 90 MMEs per day). "Chronic use" was defined as at least 10 prescriptions (regardless of MMEs) or $\geq 120$ days' supply over the 3- to 12-month post-index visit period, an outcome consistent with previous studies examining chronic use as an end point. ${ }^{28,32}$ The outcome of "opioid cessation" was defined as filling zero opioid prescriptions in months $6-12$ post-index visit. The start point for this outcome was extended from 3 months to 6 months given the clinical judgment that zero opioid medications 6 months postsurgery was a more attainable outcome for lumbar fusion.

We considered 2 sets of secondary outcomes: clinical quality indicators and costs. The assessed quality indicator outcomes were surgical complications, revision fusion, 30-day readmissions, and likelihood of discharge to home. The full list of complications and their corresponding ICD9 or CPT codes are listed in Table 1. "Thirty-day all-cause readmissions" were defined as any repeat hospitalization, regardless of cause, within 30 days of discharge from the index visit, as is concordant with previous definitions in the literature. ${ }^{8,11}$ Revision fusion was a binary variable representing the presence of any subsequent lumbar fusion 
TABLE 1. Procedures, exclusion criteria, covariates, and complications

\begin{tabular}{|c|c|}
\hline Variable & ICD-9/CPT Code \\
\hline Depression & $\begin{array}{l}\text { 296.20-296.25, 296.30-296.35, } \\
300.4,311\end{array}$ \\
\hline \multicolumn{2}{|l|}{ Procedure } \\
\hline \multicolumn{2}{|l|}{ Fusion } \\
\hline Single level & $81.06-81.08$ \\
\hline Multilevel & $81.62-81.64$ \\
\hline \multicolumn{2}{|l|}{ Laminectomy } \\
\hline Single level & $03.09,630.05,630.12,630.47$ \\
\hline Multilevel & $630.17,630.48$ \\
\hline Instrumentation & $\begin{array}{l}228.40,228.41,228.42,228.43, \\
228.44,228.45,228.46,228.47, \\
228.48\end{array}$ \\
\hline Revision fusion & $\begin{array}{l}813.6,813.7,813.8,228.50,228.52, \\
228.55\end{array}$ \\
\hline Spondylolisthesis & $756.12,738.4$ \\
\hline \multicolumn{2}{|l|}{ Exclusion criterion } \\
\hline Trauma & $\begin{array}{l}80.50-80.59,83.90-83.95 \\
95.20-95.24,95.28,95.29\end{array}$ \\
\hline Postlaminectomy syndrome & $722.81-722.83$ \\
\hline Cancer & $140-172,174-195.8,200-208,238.6$ \\
\hline Paralysis & $\begin{array}{l}334.1,342 . x, 343 . x, 344 . x, 438.2 \\
438.3,438.4,438.5,780.72\end{array}$ \\
\hline Schizophrenia & 295 \\
\hline Bipolar disorder & $296.4,296.6,296.7$ \\
\hline \multicolumn{2}{|l|}{ Comorbidity } \\
\hline Anxiety & $300.00-300.02,309.24$ \\
\hline COPD & $\begin{array}{l}416.8,416.9,490-505,506.4,508.1 \\
508.8\end{array}$ \\
\hline $\mathrm{CHF}$ & $\begin{array}{l}398.91,402.01,402.11,402.91 \\
404.01,404.03,404.11,404.13 \\
404.91,404.93,425.4-425.9,428\end{array}$ \\
\hline Hypertension & $401,402-405,642,437.2$ \\
\hline Ml/ischemic heart disease & $410,412,998.0,9971,411,429.7$ \\
\hline Diabetes, uncomplicated & $250.0-250.3,250.7$ \\
\hline Diabetes, complicated & $250.4-250.6$ \\
\hline Obesity & $\begin{array}{l}278.0,649.1,793.91, \text { V853, V854, } \\
\text { V8554 }\end{array}$ \\
\hline Drug abuse & $\begin{array}{l}292.0,292.8-292.9,304,305.2- \\
305.9,648.3\end{array}$ \\
\hline Alcohol abuse & $\begin{array}{l}265.2,291.1-291.3,291.5,291.8,303 \\
305.0\end{array}$ \\
\hline Tobacco use & V15.82, $989.84,649.0$ \\
\hline Treatment for tobacco use* & $\begin{array}{l}\text { 99406, 99407, S9075, S9453, } \\
\text { 99381-99397, 96150-96155, } 99078\end{array}$ \\
\hline \multicolumn{2}{|l|}{ Complication } \\
\hline Infection & $998.5,998.0,999.3$ \\
\hline Infection* & $\begin{array}{l}10060,10140,10180,12020,12021, \\
20005,21501,22010,22015\end{array}$ \\
\hline Chronic pain (new) & $338.2,338.4$ \\
\hline Thromboembolism & $453.0,453.4,453.8,453.2,453.3$ \\
\hline DVT & $451,453.4,453.8,453.2,453.1,453.9$ \\
\hline
\end{tabular}

» CONTINUED FROM PREVIOUS COLUMN

TABLE 1. Procedures, exclusion criteria, covariates, and complications

\begin{tabular}{ll}
\hline \multicolumn{1}{c}{ Variable } & \multicolumn{1}{c}{ ICD-9/CPT Code } \\
\hline $\begin{array}{c}\text { Complication (continued) } \\
\text { Pulmonary embolism }\end{array}$ & 415.1 \\
\hline $\begin{array}{l}\text { Wound dehiscence or } \\
\text { disruption }\end{array}$ & 998.3 \\
\hline Wound hematoma & 998.1 \\
\hline Other wound complication & $998.3,998.81,998.83,998.4$ \\
\hline $\begin{array}{c}\text { Other pulmonary complica- } \\
\text { tion }\end{array}$ & $997.3,518.4,518.5,518.7,518.81$, \\
\hline $\begin{array}{c}\text { General neurological } \\
\text { complication }\end{array}$ & $518.82,518.83,518.84,518.89,519.1$ \\
\hline Dysrhythmia & $430,431,432,433,434,435,436$, \\
& $427,426.1,426,426.3,426.4,426.5$, \\
& $426.6,426.7,426.8$ \\
\hline
\end{tabular}

$\mathrm{CHF}=$ congestive heart failure; $\mathrm{COPD}=$ chronic obstructive pulmonary disease; DVT = deep vein thrombosis; $\mathrm{MI}=$ myocardial infarction.

* Indicates corresponding CPT codes; all other codes are ICD-9 codes.

(using the same ICD-9 and CPT codes used to define the original procedure) within the coverage period. Similarly, discharge home was a binary variable indicating whether the patient was discharged to home after the initial fusion surgery (rather than to a skilled nursing or rehabilitation facility, hospice, and so forth), which is coded as a categorical variable in the MarketScan database representing location of discharge.

The secondary economic outcomes were total costs 1 and 2 years after surgery, after excluding any negative or zero-valued payments. The logarithm of this cost was used in regression modeling to account for the right-skewed nature of the outcome.

\section{Exposure}

Our primary independent variable of interest was a diagnosis of depression prior to surgery. We considered patients to have carried a preoperative diagnosis of depression if they had at least 1 recorded ICD-9 code corresponding to a diagnosis of depression in the 6 months prior to their surgery, a definition that has been shown to have a $99.66 \%$ specificity for identifying "true" cases of depression (identified using manual chart review). ${ }^{12}$ The high specificity of this particular definition was desirable given the large cohort size and the desire to restrict our depression subgroup to true cases to decrease the likelihood of false-positive associations. A full list of the ICD-9 codes used to define depression diagnosis can be found in Table 1.

\section{Covariates}

To control for differences in demographic variables and comorbidities in patients with and without depression, the full set of covariates listed in Table 1 were included in all models. This list included procedure characteristics (single-level vs multilevel fusion, single-level vs multilevel laminectomy, instrumentation), demographic information (age, sex, geographic region, year of surgery), and comorbidities (anxiety, chronic obstructive pulmonary disease, 
congestive heart failure, hypertension, ischemic heart disease, uncomplicated diabetes, complicated diabetes, obesity, drug abuse, alcohol abuse, tobacco use, and treatment for tobacco use). Age was defined as a categorical variable roughly corresponding to decade of life (18-34, 35-44, $45-54,55-64$, and $\geq 65$ years). All comorbidities were defined as the presence of at least 1 associated ICD-9 code in the patient's chart at any point prior to surgery.

To adequately represent other patient characteristics that have been shown to influence outcomes (particularly prescription drug-related outcomes), such as benzodiazepine use and preoperative opioid use, ${ }^{32}$ we also included preoperative opioid, benzodiazepine, and antipsychotic use as covariates in all regression models (coded as binary variables representing the presence or absence of at least 1 prescription for the medication class in the year preceding the index surgery). Given the particularly strong association between preoperative opioid use and adverse surgical outcomes, ${ }^{19,28}$ preoperative opioid use was coded as a 3-level variable denoting zero, low, or high usage in the 6 months prior to surgery. "Zero usage" was defined as filling 0 prescriptions for opioid medications in the 6 months prior to the index hospitalization, whereas "low usage" was defined as nonzero usage that, when ranked as a percentile in terms of MME use compared with the rest of the cohort, fell below the top 25\% of cumulative MME dose, also in the 6 months preceding surgery. Patients who fell in the top 25\% of MMEs received in the 6 months preceding surgery were classified as having "high preoperative usage."

\section{Statistical Analyses}

We used logistic regression to examine the relationship between depression status and all binary outcomes (the primary outcomes of chronic opioid use and opioid cessation, as well as the secondary outcomes of complications, 30-day readmissions, revision fusion, and discharge home). Negative binomial regression was performed to predict the counts of cumulative MMEs received months 3-12 postoperation, and a log-linear regression model was generated to predict log total cost at 1 and 2 years after surgery. For all outcomes, the full set of demographic, comorbidity, and drug-related variables described above were included as covariates.

To assist with interpretation of the regression coefficients for cost and opioid use, we present odds ratios and confidence intervals, as well as graphs representing the predicted outcomes of a simulated patient with and without depression according to the appropriate regression models. For these graphs, we chose to simulate a patient with an age equal to the mean of the cohort and without any other comorbidities. For categorical variables (sex, geographic location), the category with the largest number of patients (female sex, residency in the southern geographic region) was selected for both depressed and nondepressed simulated patients, although this did not impact the relative difference in estimates attributable to depression. Standard errors on all plots depict the standard error of the estimate according to the full regression model, whereas differences in point estimates represent the magnitude of the effect on outcome attributable to depression. These graphs are only meant to illustrate the magnitude of the effect of depression; the significance of the marginal effect of depression can be determined using the $95 \%$ confidence interval of the odds ratio or the $p$ value of the coefficient for depression in the given tables. A $p$ value of $<0.05$ was considered significant for all outcomes. Bonferroni correction was not required, given the existence of the predefined hypothesis that depression would negatively impact all primary and secondary outcomes.

\section{Results \\ Sample Characteristics}

The exclusion criteria yielded a final sample of 60,597 patients who had undergone lumbar fusion (Fig. 1), 4985 of whom had a diagnosis of depression within 6 months of the index visit and 21,905 of whom had a diagnosis of spondylolisthesis at the time of surgery (Table 2). In comparisons between the depressed and nondepressed cohorts, there were significant differences in the percentage of women (71.7\% vs $55.6 \%$, respectively) and the mean age (53.2 vs 56.4 years, respectively). There were slight variations in the type of insurance plan between controls and depressed patients, some of which were statistically significant, although the effect sizes of these differences were small or negligible according to Hedges' g statistic. Patients with depression were significantly more likely to undergo multilevel fusion procedures $(72.3 \%$ vs $66.0 \%)$ and significantly less likely to undergo laminectomies, both single level (36.1\% vs $41.2 \%)$ and multilevel (21.7\% vs $25.8 \%$ ). Given the statistically significant differences (yet small effect sizes) between the groups, all of the above variables were included as covariates in the final regression models, as were the other comorbidities and drug-use variables described in Methods. Of the 21,905 patients with a diagnosis of spondylolisthesis at the time of surgery, 1675 had a diagnosis of depression. The difference in the prevalence of spondylolisthesis in the control group versus the depressed group was negligible in magnitude according to Hedges' g statistic (36.3\% vs $33.6 \%$ ).

Postoperatively, the patients with depression received higher doses of opioid medications, and $31.8 \%$ of patients with depression met the criteria for having chronic opioid use as compared with $18.4 \%$ of controls. A greater proportion of patients without depression received zero opioids 6-12 months postsurgery (57.9\% vs $44.1 \%)$. Moreover, the patients with depression experienced significantly higher rates of complications, revision fusions, and 30-day readmissions, although the magnitude of the differences was small. There was no significant difference in the likelihood of being discharged home following surgery. The average 1-year per-patient total costs (including index visit) were $\$ 66,376$ in controls and $\$ 73,467$ in the depressed patients. At 2 years postfusion, the average total cost increased to $\$ 80,257$ for controls and $\$ 94,150$ for depressed patients.

\section{Primary Opioid Outcomes}

Opioid use after surgery varied widely depending on depression diagnosis and preoperative opioid use. Depres- 


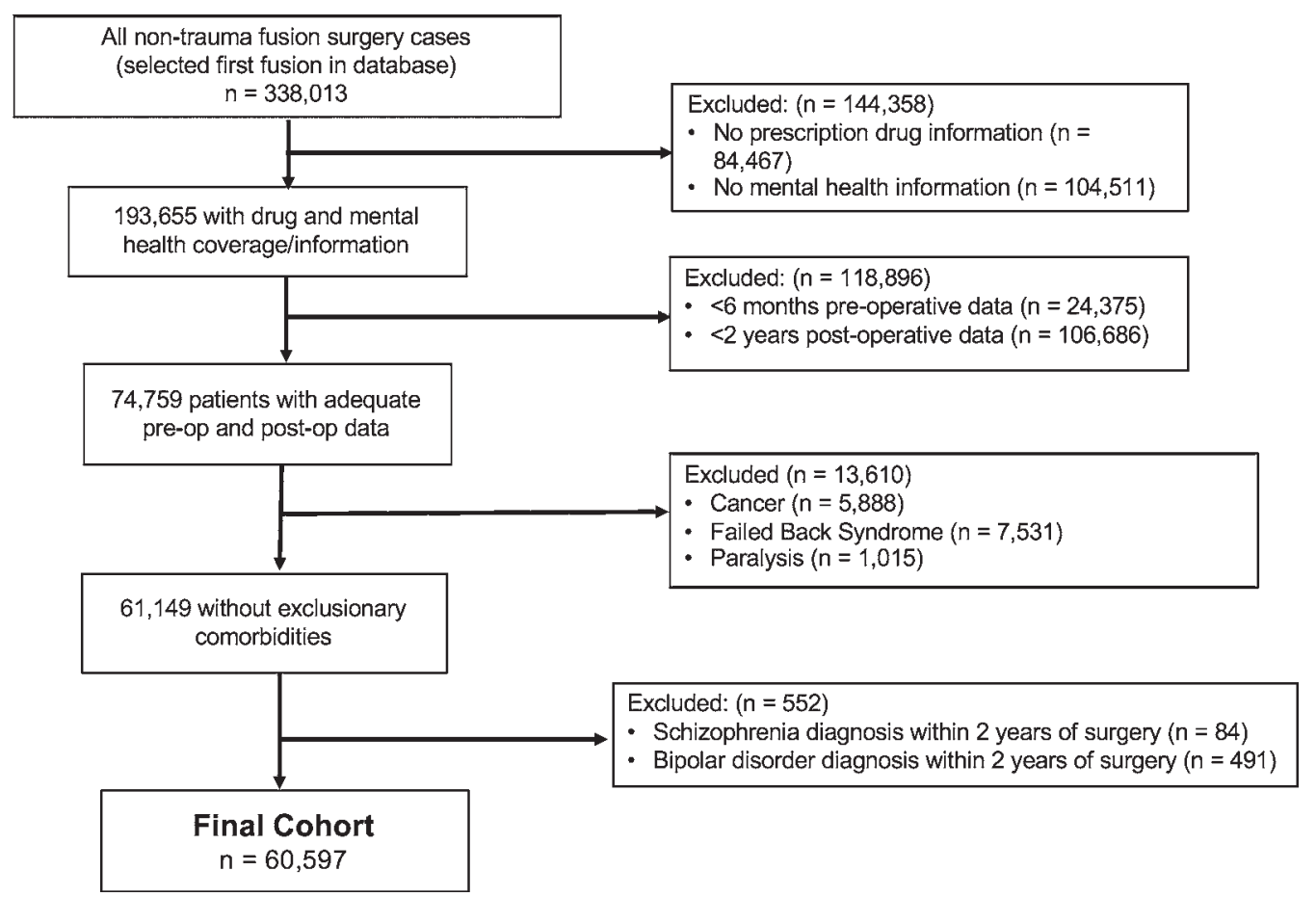

FIG. 1. Flow diagram of criteria for patient inclusion. Note that many participants fell into more than 1 category per box on the right side of the diagram. For example, some participants were lacking both prescription drug information and mental health information; therefore, they appear in each of those separate categories but were counted only once in the total number excluded.

sion diagnosis was associated with increased cumulative opioid use (measured in MMEs) 3-12 months after surgery $(\beta=0.25, p<0.001$; Table 3$)$, controlling for the covariates specified above. The effect of preoperative opioid use was even larger, yet the effect of depression remained after controlling for preoperative opioid use (Fig. 2 and Table 4). These relationships are apparent in the graph depicting the predicted total opioid use of a hypothetical patient with different levels of preoperative opioid consumption based on the negative binomial regression model. While a depression diagnosis increased the predicted postsurgical MMEs (months 3-12 postoperatively) of an otherwise healthy opioid-naïve patient from 612.9 to 787.9 (Fig. 2), having high preoperative use increased this estimate to $10,224.4$ (without depression) or 13,145.9 (with depression).

Similar patterns were observed with respect to chronic opioid use, defined as $\geq 10$ prescriptions or $\geq 120$ days' supply in the 3-12 months after surgery. A depression diagnosis was associated with significantly increased rates of chronic postoperative opioid use, even after controlling for preoperative opioid use (adjusted OR 1.28, 95\% CI 1.17-1.40; Fig. 3). In an otherwise healthy patient with high preoperative use, depression increased the estimated probability of chronic use from $53.2 \%$ to $59.2 \%$ (Fig. 2). In a similar patient with no preoperative usage, depression increased the predicted probability of chronic opioid use from $4.4 \%$ to $5.6 \%$.

Depression was also associated with a decreased probability of opioid cessation during postoperative months 6-12. Again, however, preoperative use was a strong negative driver of this outcome, yet the effect of depression remained after controlling for this variable (OR $0.96,95 \%$ CI 0.95-0.98; Fig. 3). To illustrate this, Fig. 2 depicts the overall predicted probability of a patient ceasing opiate use. With zero preoperative opioid use, the predicted probability of receiving zero opioid prescriptions from months 6 to 12 postoperatively decreased slightly less than $4 \%$ with a diagnosis of depression (from $74.1 \%$ without depression to $70.4 \%$ with depression). The predicted probability of cessation in a patient with heavy preoperative opiate use was much lower (only $21.0 \%$ and $24.7 \%$ with and without comorbid depression, respectively).

\section{Secondary Quality Indicator and Cost Outcomes}

Overall, a diagnosis of depression within the 6 months before surgery was associated with increased rates of complications (OR 1.14, 95\% CI 1.03-1.25), revisions (OR $1.15,95 \%$ CI 1.05-1.26), and 30-day readmissions (OR $1.19,95 \%$ CI 1.04-1.36) after controlling for demographic, comorbidity, and preoperative drug-use variables (Fig. 3). The association between depression and complications remained even after controlling for revision surgeries (OR $1.13,95 \%$ CI 1.03-1.26). Preoperative depression did not influence the likelihood of being discharged home after surgery after controlling for these same covariates (OR $0.92,95 \%$ CI $0.84-1.01$ ).

Diagnosis of depression was also correlated with increased 1- and 2-year costs. A depression diagnosis in an otherwise healthy patient was associated with an estimated \$3024 increase in total 1-year costs, including the cost of the initial procedure (log-linear regression, $\beta=0.06, \mathrm{p}$ $<0.001$; Table 3 and Fig. 4). The estimated cost increase 
TABLE 2. Characteristics of patients with and without depression

\begin{tabular}{|c|c|c|c|c|}
\hline Variable & No Depression Diagnosis & Preop Depression & p Value ${ }^{*}$ & Hedges' g \\
\hline No. of cases & 55,612 & 4,985 & & \\
\hline Mean age in yrs & $56.4 \pm 12.6$ & $53.2 \pm 11.2$ & $<0.001$ & 0.26 \\
\hline Male & $24,693(44.4 \%)$ & $1,412(28.3 \%)$ & $<0.001$ & 0.33 \\
\hline \multicolumn{5}{|l|}{ Region } \\
\hline Northeast & $8,379(15.1 \%)$ & $997(20.0 \%)$ & $<0.001$ & -0.136 \\
\hline North central & $17,024(30.6 \%)$ & $1,482(29.7 \%)$ & 0.200 & 0.019 \\
\hline South & $20,719(37.3 \%)$ & $1,588(31.8 \%)$ & $<0.001$ & 0.11 \\
\hline West & $8,991(16.2 \%)$ & $861(17.3 \%)$ & 0.045 & -0.03 \\
\hline Unknown & $499(0.9 \%)$ & $57(1.1 \%)$ & 0.0952 & -0.03 \\
\hline \multicolumn{5}{|l|}{ Insurance plan type } \\
\hline Comprehensive & $9,054(16.3 \%)$ & $485(9.7 \%)$ & $<0.001$ & 0.18 \\
\hline EPO & $355(0.6 \%)$ & $19(0.4 \%)$ & 0.0334 & 0.03 \\
\hline HMO & $7,676(13.8 \%)$ & $775(15.5 \%)$ & $<0.001$ & -0.05 \\
\hline POS & $4,519(8.1 \%)$ & $355(7.1 \%)$ & 0.013 & 0.04 \\
\hline PPO & $27,346(49.2 \%)$ & $2,508(50.3 \%)$ & 0.13 & 0.02 \\
\hline POS with capitation & $111(0.2 \%)$ & $9(0.2 \%)$ & 0.90 & 0.004 \\
\hline CHDP & $1,652(3.0 \%)$ & $150(3.0 \%)$ & 0.91 & 0.002 \\
\hline HDHP & $677(1.2 \%)$ & $62(1.2 \%)$ & 0.92 & 0.002 \\
\hline Unknown & $4,222(7.6 \%)$ & $622(12.5 \%)$ & $<0.001$ & -0.18 \\
\hline \multicolumn{5}{|l|}{ Procedure characteristic } \\
\hline Multilevel fusion & $36,680(66.0 \%)$ & $3,602(72.3 \%)$ & $<0.001$ & -0.13 \\
\hline \multicolumn{5}{|l|}{ Laminectomy } \\
\hline Single level & $22,939(41.2 \%)$ & $1,802(36.1 \%)$ & $<0.001$ & 0.10 \\
\hline Multilevel & $14,333(25.8 \%)$ & $1,081(21.7 \%)$ & $<0.001$ & 0.09 \\
\hline Instrumentation & $46,898(84.3 \%)$ & $4,230(84.9 \%)$ & 0.339 & -0.014 \\
\hline \multicolumn{5}{|l|}{ Primary outcome } \\
\hline Mean cumulative dose in MMEs & $4042.4 \pm 14760.9$ & $8761.6 \pm 22,243.9$ & $<0.001$ & -0.30 \\
\hline Chronic postop opiate use & $10,245(18.4 \%)$ & $1,585(31.8 \%)$ & $<0.001$ & -0.34 \\
\hline Opiate cessation mos 6-12 & $32,187(57.9 \%)$ & $2,200(44.1 \%)$ & $<0.001$ & 0.28 \\
\hline \multicolumn{5}{|l|}{ Secondary outcome } \\
\hline Complications & $6,770(12.2 \%)$ & $707(14.2 \%)$ & $<0.001$ & -0.06 \\
\hline Revision fusion (any) & $6,966(12.5 \%)$ & $763(15.3 \%)$ & $<0.001$ & -0.08 \\
\hline 30-day readmission & $2,686(4.8 \%)$ & $335(6.7 \%)$ & $<0.001$ & -0.09 \\
\hline Discharged home & $43,755(78.7 \%)$ & $3,935(78.9 \%)$ & 0.684 & -0.01 \\
\hline Mean 1-yr costs & $\$ 66,376 \pm 60,822$ & $\$ 73,467 \pm 61,657$ & $<0.001$ & -0.12 \\
\hline Mean 2-yr costs & $\$ 80,257 \pm 77,959$ & $\$ 94,150 \pm 85,268$ & $<0.001$ & -0.18 \\
\hline Spondylolisthesis diagnosis & $20,230(36.3 \%)$ & $1,675(33.6 \%)$ & $<0.001$ & -0.06 \\
\hline
\end{tabular}

$\mathrm{CHDP}=$ Child Health and Disability Prevention program; $\mathrm{EPO}=$ exclusive provider organization; HDHP = high-deductible health plan; HMO = health maintenance organization; $\mathrm{POS}=$ point of service; $\mathrm{PPO}=$ preferred provider organization.

Values expressed as the mean \pm standard deviation or as number (\%) unless otherwise stated.

* Denotes $p$ value of t-test (continuous variables) or chi-square test (categorical variables); standardized effect size calculated using Cohen's d with Hedges' $g$ correction.

attributable to depression was $\$ 5598$ at 2 years post-index surgery, again controlling for all previously described demographic, comorbidity, and preoperative drug variables (log-linear regression, $\beta=0.09, \mathrm{p}<0.001$ ).

\section{Discussion}

In the present study, we sought to examine the effects of a preoperative diagnosis of depression on a variety of functional outcomes following lumbar fusion surgery using a nationwide administrative sample. We found that a diagnosis of depression prior to lumbar fusion was associated with increased postoperative opioid use, even after controlling for preoperative opioid use, demographic factors, and relevant comorbidities. In addition, preoperative depression was associated with increased rates of complications, revisions, and 30-day readmissions, as well as increased 1- and 2-year costs. Depression diagnosis was not 
TABLE 3. Effect of preoperative depression on cumulative opioid use and 1- and 2-year costs

\begin{tabular}{ccc}
\hline Outcome & $\beta$ & $p$ Value \\
\hline Primary & & \\
\hline Cumulative MMEs & 0.25 & $<0.001$ \\
\hline Secondary & & \\
\hline 1-yr total cost & 0.06 & $<0.001$ \\
\hline 2-yr total cost & 0.09 & $<0.001$ \\
\hline
\end{tabular}

$\beta$ indicates the coefficient of depression in the multivariable negative binomial and log-linear regression models predicting cumulative MMEs and total costs at 1 and 2 years after fusion surgery. The $p$ values indicate the significance of the marginal effect of depression while controlling for the full set of demographic, comorbidity, and drug-use variables detailed in the text.

significantly associated with the likelihood of discharge to home after lumbar fusion surgery.

While previous research has implicated preoperative depression in adverse outcomes of lumbar fusion surgery, ${ }^{4,6,24}$ our study is the first, to our knowledge, to investigate this topic using a nationwide administrative database containing records from privately insured individuals and patients with Medicare. The consistent nature of this relationship between depression and adverse outcomes supports the notion of depression as a disease affecting both physical and mental health as well as health care utilization. The association between depression and both 1- and 2-year costs highlights the fact that the economic burden of depression, while well established in the general population, ${ }^{13}$ plays an important role in the long-term costs after
TABLE 4. Effect of preoperative opioid use on opioid outcomes

\begin{tabular}{|c|c|c|c|c|}
\hline Outcome & OR & $95 \% \mathrm{Cl}$ & $\beta$ & $\mathrm{p}$ Value \\
\hline \multicolumn{5}{|l|}{ Total MMEs } \\
\hline Low preop usage (vs none) & & & 0.67 & $<0.001$ \\
\hline High preop usage (vs none) & & & 2.81 & $<0.001$ \\
\hline \multicolumn{5}{|l|}{ Opiate cessation mos 6-12 } \\
\hline Low preop usage (vs none) & 0.85 & $0.84-0.85$ & & \\
\hline High preop usage (vs none) & 0.61 & $0.60-0.62$ & & \\
\hline \multicolumn{5}{|l|}{ Chronic use mos 3-12 } \\
\hline Low preop usage (vs none) & 2.53 & $2.34-2.75$ & & \\
\hline High preop usage (vs none) & 24.53 & $22.54-26.34$ & & \\
\hline
\end{tabular}

$\beta$ indicates the coefficient of preoperative use in the negative binomial regression model predicting cumulative MMEs postoperatively. Odds ratios represent the adjusted odds ratios when predicting postoperative chronic use months 3-12 postoperatively and opioid cessation months $6-12$ postoperatively. The $p$ values correspond to the significance of the marginal effect of depression on the outcome of interest.

lumbar fusion. While the administrative nature of data in the current study precluded us from assessing the treatment status of patients prior to surgery, in the future it would be worthwhile to determine whether preoperative treatment of depression would improve outcomes. Given the high prevalence of spondylolisthesis in this cohort that underwent lumbar fusion, future research investigating the specific effect of depression diagnosis within this subgroup would be of great utility.

Opioid use is common following lumbar fusion, and the
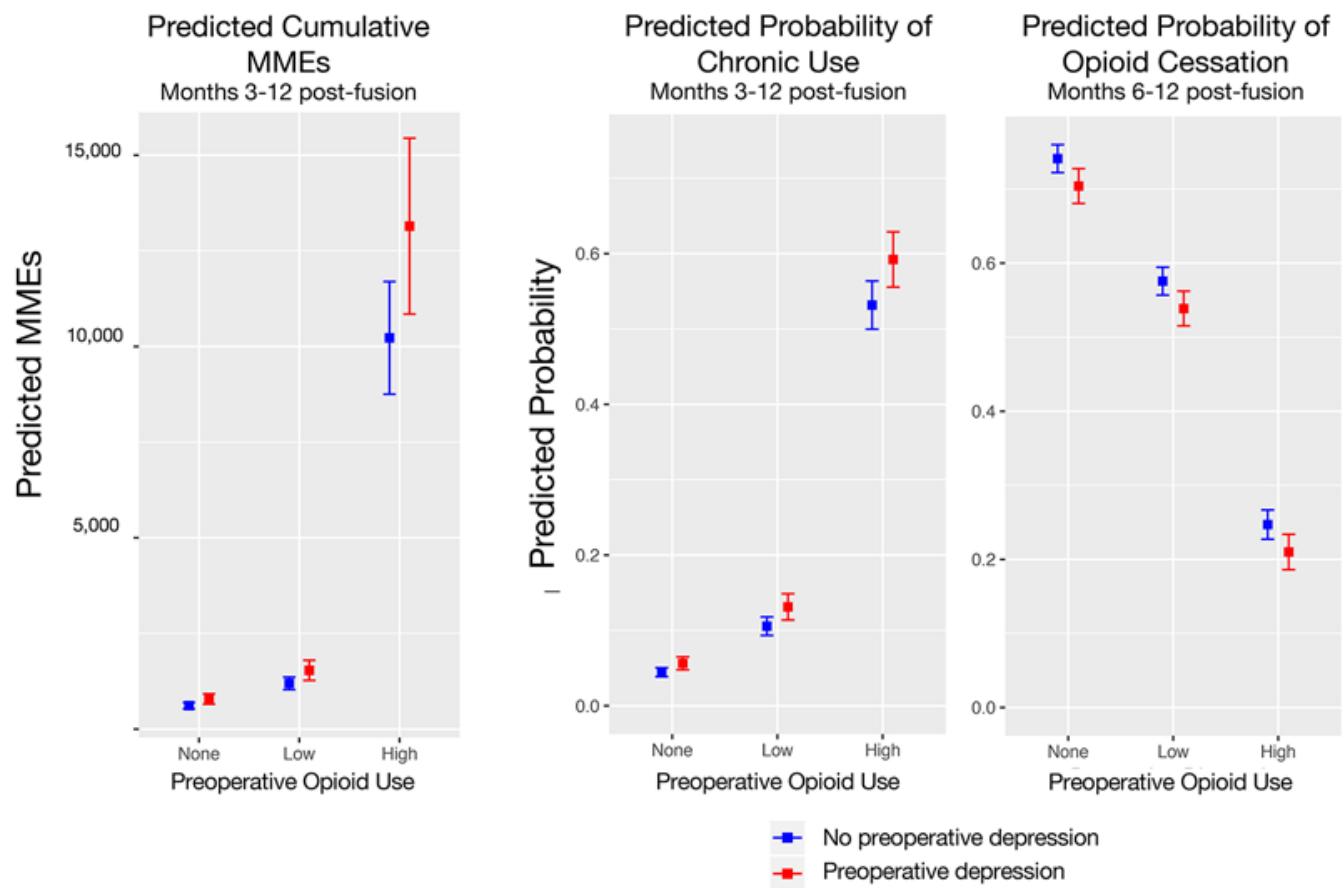

FIG. 2. Opioid outcomes according to depression diagnosis, stratified by preoperative opioid use. The predicted postoperative opioid use of an otherwise healthy patient with various levels of preoperative opioid use, both with and without depression, was calculated using the final logistic and negative binomial regression models. Error bars indicate the standard error of the overall estimate (estimated probability or cumulative MMEs) using multivariable logistic or negative binomial regression. 


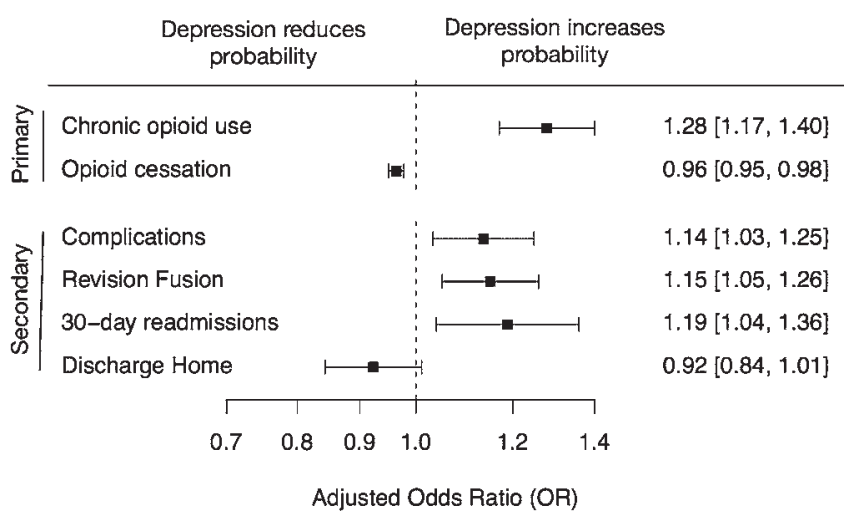

FIG. 3. Adjusted odds ratios of preoperative depression for binary outcomes. All odds ratios were adjusted for the full set of demographic, comorbidity, and drug-use variables detailed in the text.

rising rate of opioid abuse in recent years has become a growing problem in this country. ${ }^{30}$ After controlling for demographic factors and comorbidities, we found that a preoperative depression diagnosis was associated with increased cumulative MMEs and risk of chronic use 3-12 months after surgery, as well as a decreased probability of opioid cessation 6-12 months postoperatively. Previous studies have identified depression among the numerous factors increasing the risk of opioid use after surgeries such as total hip arthroplasty and bariatric surgery; 9,27 however, its role in lumbar fusion has remained unexplored. Our results suggest that depression may be an important driver of postoperative opioid use in this surgical population, even after controlling for preoperative use. This relationship between depression and postoperative opioid use, particularly in patients with a diagnosis of spondylolisthesis, warrants further investigation.

\section{Study Limitations}

Results of the present study must be interpreted con- sidering the limitations inherent to all large database studies. Specifically, our categorization of depression diagnosis, along with comorbidities and outcomes, assumed the accuracy of our administrative data. Although we used a definition of depression that was based on a previously validated report, the incidence of depression in administrative data based on ICD-9 codes may underestimate the true prevalence. ${ }^{12}$ Additionally, depression is commonly thought to be a spectrum of disorders rather than one distinct entity; therefore, characterizing it with one group of ICD-9 codes may not achieve the granularity necessary to distinguish between different forms of the disorder.

While generalized anxiety or panic disorder was included as a covariate in our analysis, other psychiatric conditions, such as Axis II disorders and other mood disorders, particularly bipolar disorder and other anxiety disorders, are frequently comorbid with depression and may affect the relationship between depression and outcomes. ${ }^{20}$ Additionally, we did not control for health care utilization, which has been associated with a diagnosis of depression as well. ${ }^{10}$ Further work is required to investigate the nature of this relationship and its effect on the association between depression and lumbar fusion outcomes.

Finally, opioid use was assessed based on the number and dosage of medications that were both prescribed to the patient and picked up at a pharmacy, not on actual patient ingestion, which may not reflect opioids obtained from other sources not covered by insurance. To more definitively characterize the relationship between depression and opioid use, future studies using more granular data on both depression diagnosis and opioid use would be of great value.

\section{Conclusions}

Overall, the current study provides insight into the relationship between preoperative depression and a variety of outcomes, particularly opioid use, following lumbar fusion. Given that depression is a potentially modifiable risk factor for adverse outcomes, future work might identify

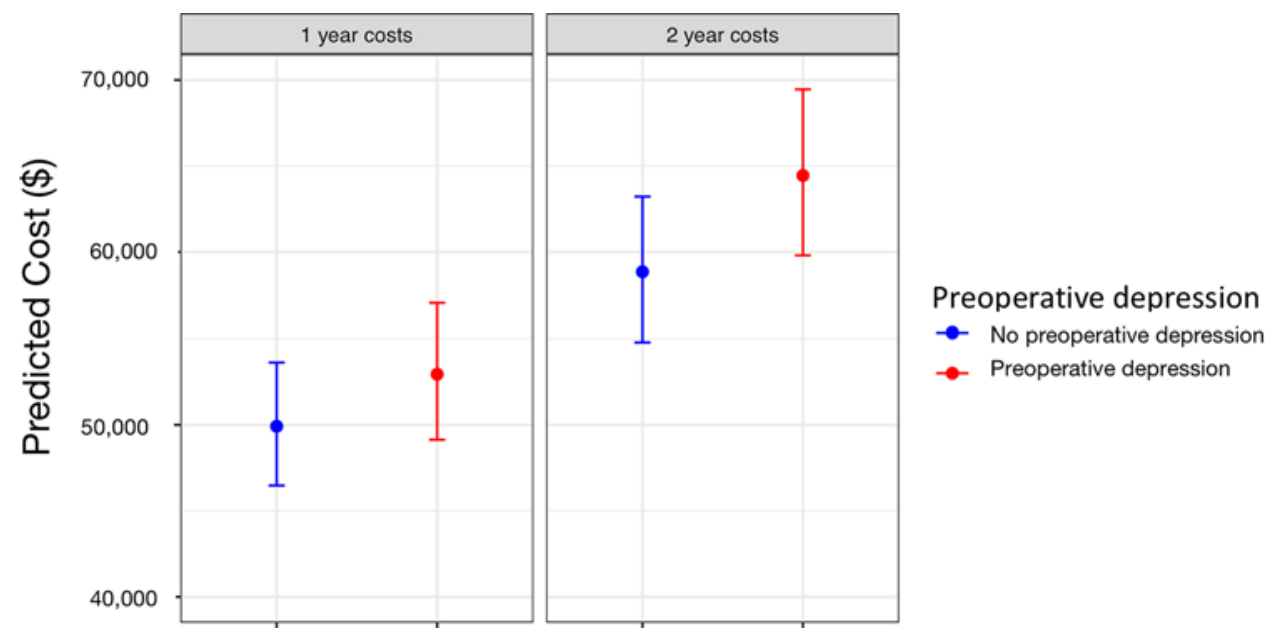

FIG. 4. Predicted 1-year and 2-year costs, according to depression diagnosis. Costs were calculated with the log-linear regression model predicting 1 - and 2-year costs using a hypothetical patient with an age equal to the mean of the sample and no other comorbidities. Total 1-year and 2-year predicted log costs were exponentiated to calculate the actual predicted cost. Error bars indicate the standard error of the overall estimate using log-linear regression; these overlapped even when the marginal effect of depression was statistically significant due to the combined uncertainty of other coefficients in the model. 
ways to mitigate the impact of depression and to improve outcomes for patients undergoing lumbar fusion. In assessing postfusion outcomes, we believe that the preoperative assessment of depression should be included and that the disorder should be considered as a potentially impactful comorbid condition.

\section{Acknowledgments}

We thank Keith Humphreys for his insightful comments and contributions to this manuscript.

\section{References}

1. Aalto TJ, Malmivaara A, Kovacs F, Herno A, Alen M, Salmi L, et al: Preoperative predictors for postoperative clinical outcome in lumbar spinal stenosis: systematic review. Spine (Phila Pa 1976) 31:E648-E663, 2006

2. Adogwa O, Carr K, Fatemi P, Verla T, Gazcon G, Gottfried $\mathrm{O}$, et al: Psychosocial factors and surgical outcomes: are elderly depressed patients less satisfied with surgery? Spine (Phila Pa 1976) 39:1614-1619, 2014

3. Adogwa O, Elsamadicy AA, Mehta AI, Vasquez RA, Cheng J, Karikari IO, et al: Association between baseline affective disorders and 30-day readmission rates in patients undergoing elective spine surgery. World Neurosurg 94:432-436, 2016

4. Alentado VJ, Caldwell S, Gould HP, Steinmetz MP, Benzel EC, Mroz TE: Independent predictors of a clinically significant improvement after lumbar fusion surgery. Spine J 17:236-243, 2017

5. Ananthakrishnan AN, Gainer VS, Perez RG, Cai T, Cheng SC, Savova G, et al: Psychiatric co-morbidity is associated with increased risk of surgery in Crohn's disease. Aliment Pharmacol Ther 37:445-454, 2013

6. Anderson JT, Haas AR, Percy R, Woods ST, Ahn UM, Ahn NU: Clinical depression is a strong predictor of poor lumbar fusion outcomes among workers' compensation subjects. Spine (Phila Pa 1976) 40:748-756, 2015

7. Arnow BA, Hunkeler EM, Blasey CM, Lee J, Constantino MJ, Fireman B, et al: Comorbid depression, chronic pain, and disability in primary care. Psychosom Med 68:262-268, 2006

8. Arundel C, Lam PH, Khosla R, Blackman MR, Fonarow GC, Morgan C, et al: Association of 30-day all-cause readmission with long-term outcomes in hospitalized older Medicare beneficiaries with heart failure. Am J Med 129:1178-1184, 2016

9. Clarke H, Soneji N, Ko DT, Yun L, Wijeysundera DN: Rates and risk factors for prolonged opioid use after major surgery: population based cohort study. BMJ 348:g1251, 2014

10. Dennehy EB, Robinson RL, Stephenson JJ, Faries D, Grabner M, Palli SR, et al: Impact of non-remission of depression on costs and resource utilization: from the COmorbidities and symptoms of DEpression (CODE) study. Curr Med Res Opin 31:1165-1177, 2015

11. Elsamadicy AA, Adogwa O, Vuong VD, Mehta AI, Vasquez RA, Cheng J, et al: Patient body mass index is an independent predictor of 30-day hospital readmission after elective spine surgery. World Neurosurg 96:148-151, 2016

12. Fiest KM, Jette N, Quan H, St Germaine-Smith C, Metcalfe A, Patten SB, et al: Systematic review and assessment of validated case definitions for depression in administrative data. BMC Psychiatry 14:289, 2014

13. Greenberg PE, Fournier AA, Sisitsky T, Pike CT, Kessler RC: The economic burden of adults with major depressive disorder in the United States (2005 and 2010). J Clin Psychiatry 76:155-162, 2015

14. Gum JL, Glassman SD, Carreon LY: Is type of compensation a predictor of outcome after lumbar fusion? Spine (Phila Pa 1976) 38:443-448, 2013

15. Kanouse AB, Compton P: The epidemic of prescription opioid abuse, the subsequent rising prevalence of heroin use, and the federal response. J Pain Palliat Care Pharmacother 29:102-114, 2015

16. Kupfer DJ, Frank E, Phillips ML: Major depressive disorder: new clinical, neurobiological, and treatment perspectives. Lancet 379:1045-1055, 2012

17. Manchikanti L, Giordano J, Boswell MV, Fellows B, Manchukonda R, Pampati V: Psychological factors as predictors of opioid abuse and illicit drug use in chronic pain patients. J Opioid Manag 3:89-100, 2007

18. Mark TL, Vandivort-Warren R, Miller K: Mental health spending by private insurance: implications for the mental health parity and addiction equity act. Psychiatr Serv 63:313-318, 2012

19. Martin BC, Fan MY, Edlund MJ, Devries A, Braden JB, Sullivan MD: Long-term chronic opioid therapy discontinuation rates from the TROUP study. J Gen Intern Med 26:14501457, 2011

20. Melartin TK, Rytsälä HJ, Leskelä US, Lestelä-Mielonen PS, Sokero TP, Isometsä ET: Current comorbidity of psychiatric disorders among DSM-IV major depressive disorder patients in psychiatric care in the Vantaa Depression Study. J Clin Psychiatry 63:126-134, 2002

21. Mollon B, Mahure SA, Ding DY, Zuckerman JD, Kwon YW: The influence of a history of clinical depression on perioperative outcomes in elective total shoulder arthroplasty: a ten-year national analysis. Bone Joint J 98-B:818-824, 2016

22. Nguyen TH, Randolph DC, Talmage J, Succop P, Travis R: Long-term outcomes of lumbar fusion among workers' compensation subjects: a historical cohort study. Spine (Phila Pa 1976) 36:320-331, 2011

23. Pakarinen M, Vanhanen S, Sinikallio S, Aalto T, Lehto SM, Airaksinen O, et al: Depressive burden is associated with a poorer surgical outcome among lumbar spinal stenosis patients: a 5-year follow-up study. Spine J 14:2392-2396, 2014

24. Parker SL, Godil SS, Zuckerman SL, Mendenhall SK, Devin CJ, McGirt MJ: Extent of preoperative depression is associated with return to work after lumbar fusion for spondylolisthesis. World Neurosurg 83:608-613, 2015

25. Pinheiro MB, Ferreira ML, Refshauge K, Ordoñana JR, Machado GC, Prado LR, et al: Symptoms of depression and risk of new episodes of low back pain: a systematic review and meta-analysis. Arthritis Care Res (Hoboken) 67:15911603,2015

26. Prescription Drug Monitoring Program Training and Technical Assistance Center: Technical Assistance Guide No. 01-13. Calculating Daily Morphine Milligram Equivalents. (http://www.pdmpassist.org/pdf/BJA_performance_ measure_aid_MME_conversion.pdf) [Accessed November $10,2017]$

27. Raebel MA, Newcomer SR, Bayliss EA, Boudreau D, DeBar L, Elliott TE, et al: Chronic opioid use emerging after bariatric surgery. Pharmacoepidemiol Drug Saf 23:1247-1257, 2014

28. Raebel MA, Newcomer SR, Reifler LM, Boudreau D, Elliott TE, DeBar L, et al: Chronic use of opioid medications before and after bariatric surgery. JAMA 310:1369-1376, 2013

29. Sinikallio S, Aalto T, Airaksinen O, Herno A, Kröger H, Savolainen S, et al: Depression is associated with poorer outcome of lumbar spinal stenosis surgery. Eur Spine J 16:905-912, 2007

30. Stack SJ: Confronting a crisis: an open letter to America's physicians on the opioid epidemic. Huffington Post. May 11, 2016. (https://www.huffingtonpost.com/steven-j-stack/ confronting-a-crisis-an-o_b_9911530.html) [Accessed November 8,2017$]$ 
31. Stephens JR, Steiner MJ, DeJong N, Rodean J, Hall M, Richardson T, et al: Healthcare utilization and spending for constipation in children with versus without complex chronic conditions. J Pediatr Gastroenterol Nutr 64:31-36, 2017

32. Sun EC, Darnall BD, Baker LC, Mackey S: Incidence of and risk factors for chronic opioid use among opioid-naive patients in the postoperative period. JAMA Intern Med 176:1286-1293, 2016

33. Veeravagu A, Cole TS, Jiang B, Ratliff JK, Gidwani RA: The use of bone morphogenetic protein in thoracolumbar spine procedures: analysis of the MarketScan longitudinal database. Spine J 14:2929-2937, 2014

34. Wilkerson RG, Kim HK, Windsor TA, Mareiniss DP: The opioid epidemic in the United States. Emerg Med Clin North Am 34:e1-e23, 2016

35. Wolfe S, Bouffard DL, Modesto-Lowe V: The opioid crisis and the physician's role in contributing to its resolution: Step one-Prevention of overdoses. Conn Med 80:325-334, 2016

36. Wu J, Thammakhoune J, Dai W, Koren A, Tcherny-Lessenot $\mathrm{S}, \mathrm{Wu} \mathrm{C}$, et al: Assessment of dronedarone utilization using US claims databases. Clin Ther 36:264-272, 272.e1-272.e2, 2014

\section{Disclosures}

Dr. Sun has been a consultant for Egalet Inc.

\section{Author Contributions}

Conception and design: O'Connell, Azad, Vail, Desai, Ratliff, Veeravagu. Acquisition of data: O'Connell, Azad. Analysis and interpretation of data: O'Connell, Azad, Vail, Sun. Drafting the article: O'Connell, Mittal, Johnson. Critically revising the article: O'Connell, Azad, Mittal, Vail, Johnson, Sun, Ratliff, Veeravagu. Reviewed submitted version of manuscript: Mittal, Vail, Johnson, Sun, Ratliff, Veeravagu. Statistical analysis: O'Connell. Administrative/technical/material support: Mittal, Johnson. Study supervision: Desai, Sun, Ratliff, Veeravagu.

\section{Correspondence}

Chloe O'Connell: Stanford University School of Medicine, Stanford, CA. chloeo@stanford.edu. 